\title{
Indentation-Induced Damage Mechanisms in Germanium
}

David J. Oliver ${ }^{1}$, Jodie E. Bradby ${ }^{1}$, Jim S. Williams ${ }^{1}$, Michael V. Swain ${ }^{2}$, Damien McGrouther ${ }^{3}$, and Paul Munroe ${ }^{3}$

${ }^{1}$ Electronic Materials Engineering, Australian National University, Canberra, ACT 0200, Canberrra, 0200, Australia

${ }^{2}$ Biomaterials Science Research Unit, University of Sydney, Sydney, Australia

${ }^{3}$ Electron Microscope Unit, University of New South Wales, Sydney, 2052, Australia

\begin{abstract}
The response of crystalline Ge to indentation has been studied over a range of maximum loads. At a certain load, an unusual 'giant pop-in' event occurs, in which a discontinuous extension of $>1 \mu \mathrm{m}$ is observed in the force-displacement curve. In such cases, load release curves show a pronounced 'elbowing' response, leading to increased depth recovery. TEM and Raman microspectroscopy revealed the presence of amorphous material in the residual impression. To examine cracking, a sequence of cross-sections was milled through the indent and images taken using an automated method (the 'slice-and-view' method). Using 3-D reconstruction software, the data was segmented and reconstructed into a 3-dimensional representation of the cracks around the indent. Applying this technique to indents featuring a giant pop-in, it was deduced that the inelastic elbowing observed was a bending response of material detached by lateral cracking. The giant pop-in is attributable to material removal, caused by lateral cracks formed during loading.
\end{abstract}

\section{INTRODUCTION}

Crystalline germanium (Ge) exhibits a wide range of responses to mechanical deformation with a sharp diamond indenter, including dislocation plasticity, ${ }^{1}$ twinning, ${ }^{1,2}$ phase transformations ${ }^{3}$ and at higher loads cracking. ${ }^{4,5}$ The present study has been prompted by the observation of novel features in the indentation force-displacement (P-h) curve of Ge. A sudden displacement excursion, or 'pop-in', of unusually large magnitude $(1 \mu \mathrm{m}$ or greater) was observed above a threshold load. This giant pop-in event was accompanied by a discontinuous change in slope, or 'elbow', on unloading. To understand the causes of these force-displacement features, the microstructural changes in the indent were examined using several FIB techniques, detailed below. The results indicate a material removal event is responsible for the giant pop-in, whilst the elbowing is due to the opening of lateral cracks during unloading.

\section{EXPERIMENTAL DETAILS}

Undoped crystalline Ge(100) was indented with a UMIS-2000 indenter (CSIRO), using a spheroconical diamond tip $(\mathrm{R} \approx 4.3 \mu \mathrm{m})$. Some indents were created in a single load-unload cycle; other indents were created in a multiple loading fashion, in which the load-unload cycle was 
repeated 5 times. Maximum loads ranged up to $400 \mathrm{mN}$. Loading rates of $1.0-1.5 \mathrm{mN} . \mathrm{s}^{-1}$ and unloading rates of $1.4-1.9 \mathrm{mN} . \mathrm{s}^{-1}$ were used.

Raman spectra were collected from the centers of indents using a Raman microscope (Dilor Super LabRam), using the HeNe laser line of $632.8 \mathrm{~nm}$, with a spot size of $1 \mu^{2}$. The A low laser power $(<100 \mu \mathrm{W})$ was used to avoid artifacts.

A dual-beam FIB microscope (FEI xT Nova Nanolab 200) was used to investigate subsurface damage. Thin cross-sections were prepared through the center of indents using an automated script. The cross-sections were plucked out ex situ using a Kleindiek micromanipulator, and placed on a carbon-covered copper grid for TEM examination. TEM was conducted using a Philips CM 300 operating at $300 \mathrm{kV}$.

Some indents were cross-sectioned and imaged within the FIB to examine sub-surface cracking. A $1 \mu \mathrm{m}$ strip of platinum $(\mathrm{Pt})$ was deposited to protect the area of interest, and a trench milled at a high beam current of $7 \mathrm{nA}$. Lower beam currents were then used to remove 'curtaining' features from the face of the cross-section, and then the cross-section was imaged using the electron beam, as schematised in Figure 1.

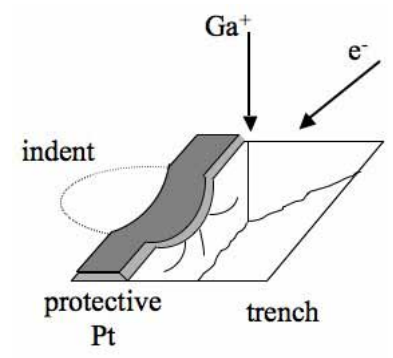

Figure 1. Schematic of cross-section geometry for viewing cracking.

Finally, a 3-dimensional representation of sub-surface cracking beneath selected indents was obtained using a FIB tomographic technique. A sequence of cross-sections through the volume of interest were milled and imaged with the FIB using an automated 'slice-and-view' script. Using the Amira (Mercury Computer Systems)software package, cracks, which showed up as darker contrast areas, were segmented using a combination of automatic and manual segmentation tools. The segmented slices were then re-combined to give a volumetric representation of the crack system.

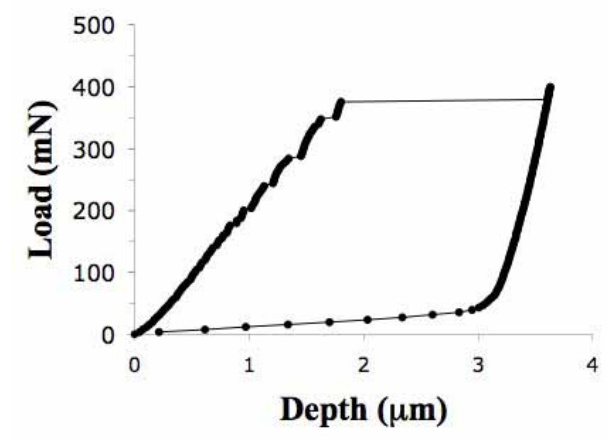

Figure 2. A $400 \mathrm{mN} \mathrm{P}$-h curve in Ge, featuring a giant pop-in. 


\section{RESULTS AND DISCUSSION}

Figure 2 shows a typical $400 \mathrm{mN}$ P-h curve for Ge with the 5 um tip. On loading, normal elasticplastic behaviour is observed at lower loads. At a threshold load of $\sim 375 \mathrm{mN}$, a sudden jump in displacement, or 'giant pop-in', of $\sim 1.8 \mu \mathrm{m}$ is observed. Unloading initially occurs with a similar slope to what would have been observed without a giant pop-in, signifying elastic recovery in the bulk. However, at low loads, below $\sim 60 \mathrm{mN}$, the slope of the unloading curve decreases sharply, so-called 'elbowing'. This enhanced compliance results in the recovery of the extra penetration caused by the giant pop-in.

Figure 3 a shows a P-h curve of 5 load-unload cycles with a maximum load of $200 \mathrm{mN}$. The first cycle shows normal elastic-plastic behaviour on loading and elastic behaviour on unloading. The second loading cycle initially follows the first unloading cycle, but deviates from elasticity at $\sim 100 \mathrm{mN}$, and a giant pop-in occurs at $\sim 130 \mathrm{mN}$. Elbowing is observed in subsequent unloading and re-loading curves, with some hysteresis between unload curves and reload curves.
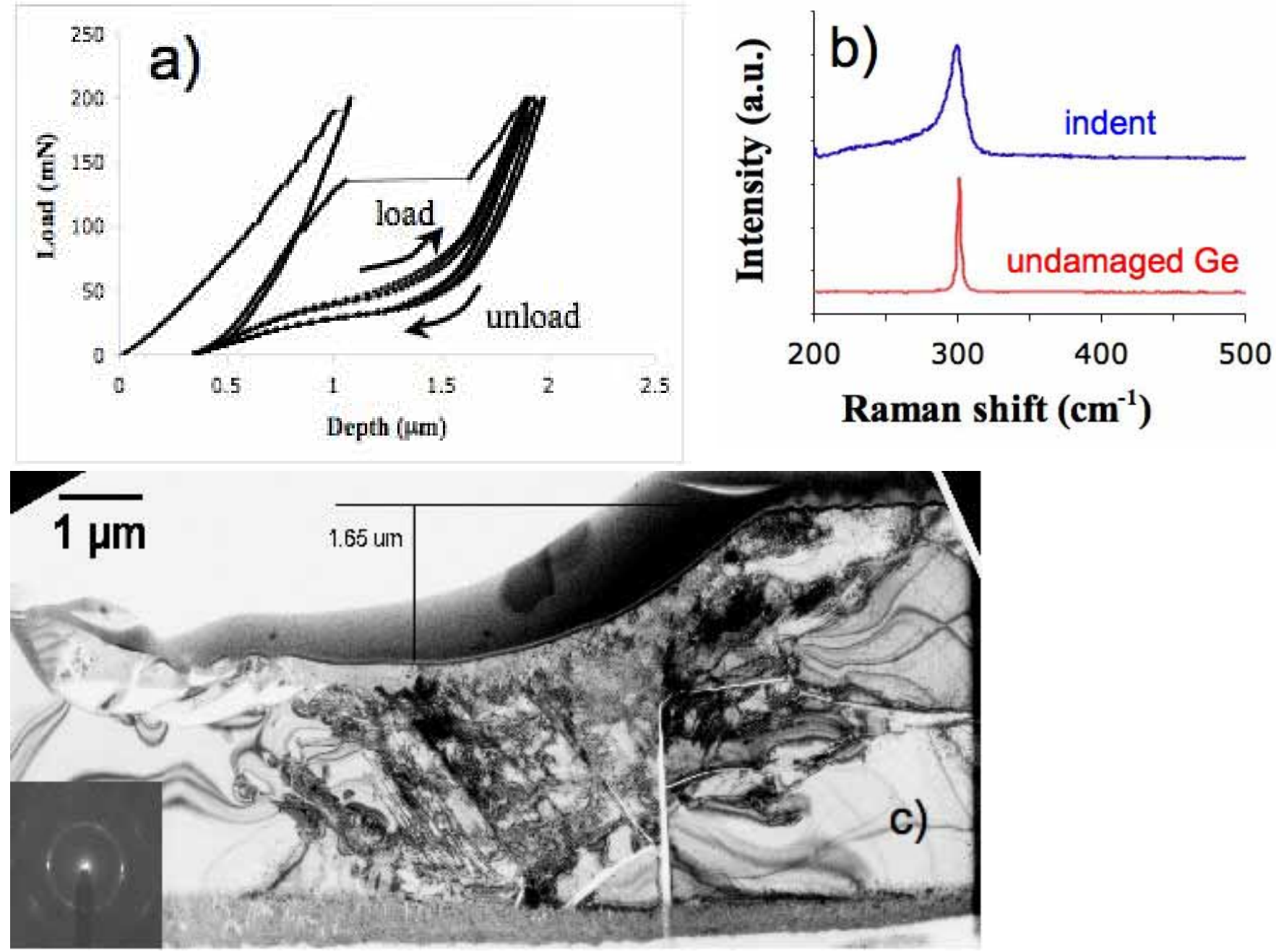

Figure 3. a) A multiple-loading ( 5 x $200 \mathrm{mN}$ ) P-h curve for Ge, featuring a giant pop-in. b) Raman spectra from the indent in a) and from undeformed Ge. c) XTEM image of the indent in a), with SADP from region directly below indent inset. 
Figure $3 \mathrm{~b}$ shows a Raman spectrum taken from the indent shown in Figure 3a, with a spectrum from undamaged $\mathrm{Ge}$ for comparison. Both spectra feature a peak at $\sim 300 \mathrm{~cm}^{-1}$ corresponding to the original diamond cubic structure. The spectrum from the indent additionally features a broad shoulder from 200 to $300 \mathrm{~cm}^{-1}$, indicating the presence of amorphous material in the indent.

Figure $3 \mathrm{c}$ shows a cross-sectional TEM micrograph of the indent. The black bands indicate slip or twin bands, as has previously been observed in Ge indents. ${ }^{1}$ Additionally, a thin region of transformed material can be observed directly beneath the surface of the indent, darker in contrast than the undamaged Ge but lighter in contrast than the slip traces. SADP (inset) indicates this transformed phase to be amorphous or nanocrystalline. Amorphous material was not observed in TEM or Raman for other indents that had not undergone a giant pop-in. This suggests that the giant pop-in somehow triggers amorphisation, although the precise mechanism is not yet clear. Amorphous material has been observed in indented Si as the product of a highpressure metallic phase transformation, ${ }^{6,7}$ but an alternative possibility in this case is that the material is the result of a crystalline-to-amorphous transition directly from the diamond cubic phase, induced by high crystal defect density. Further work is needed to explore this possibility.

The plastic impression was measured in Figure $3 \mathrm{c}$ to be $\sim 1.65 \mu \mathrm{m}$ deep, significantly greater than the depth at final unload from the P-h curve of $\sim 0.5 \mu \mathrm{m}$, which is normally assumed to coincide with the depth of the plastic impression. It appears therefore that the giant pop-in is a permanent material removal event, in which material is ejected from the volume directly beneath the indenting tip.
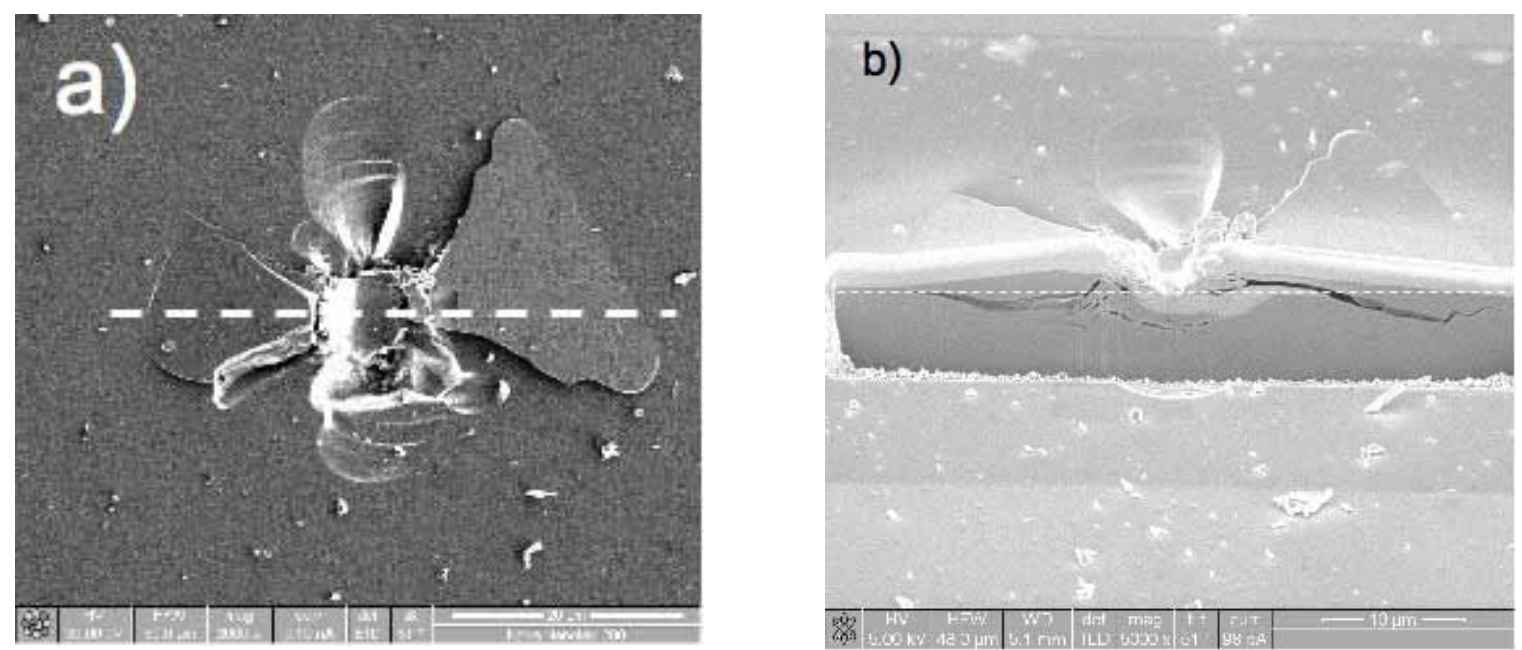

Figure 4. a) FIB image of a $350 \mathrm{mN}$ indent that underwent a giant pop-in. b) A FIB cross-section of the indent, showing sub-surface cracking.

Figure 4 shows images taken with the dual-beam FIB of an indent that underwent giant pop-in. The top-view image (Figure 4a) taken with the ion beam shows a four-fold lobe pattern of lateral cracking around the indent. Chips have been removed from two of the lobes, leaving a scalloped impression. Debris particles can be observed scattered in the vicinity of the indent. Indents with a giant pop-in were surrounded by larger quantities of debris than indents without a giant pop-in, suggesting that material is ejected onto the surface during the giant pop-in event. 
Figure $4 \mathrm{~b}$ shows a cross-sectional image taken with the electron beam. Two of the lateral cracks are clearly visible. The plates of material detached by the lateral cracks can be seen to have uplifted significantly above the original top surface. The elbowing observed in the P-h curve (Figure 2) can be attributed to the opening of the lateral cracks during unloading. The plates of material above the cracks act as cantilevers, giving rise to the observed linear and highly compliant response.

3D reconstruction obtained from slice-and-view data are shown in Figure 5. The indent is a $350 \mathrm{mN}$ indent that underwent a giant pop-in. A four-fold lateral crack system surrounds the indent. The lateral cracks curve upwards to meet the surface, allowing material to be detached and chipped away. It seems likely that this chipping is the cause of material removal, and the giant pop-in occurs when a lateral crack or cracks develops during loading.
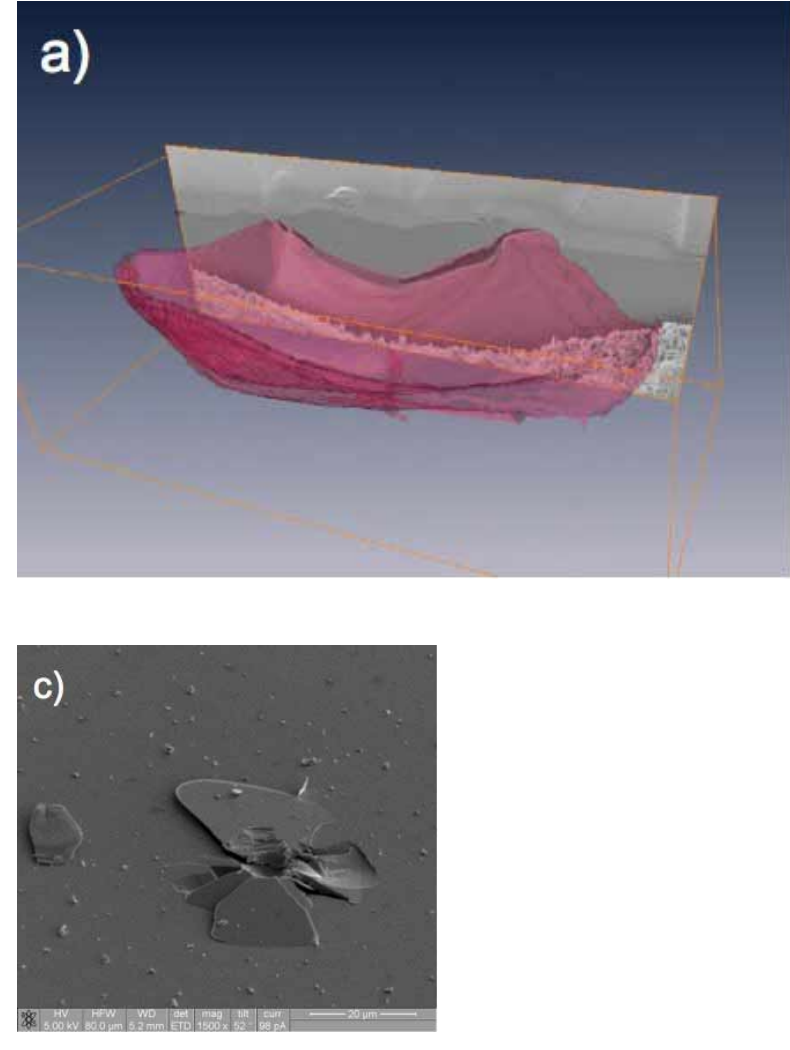

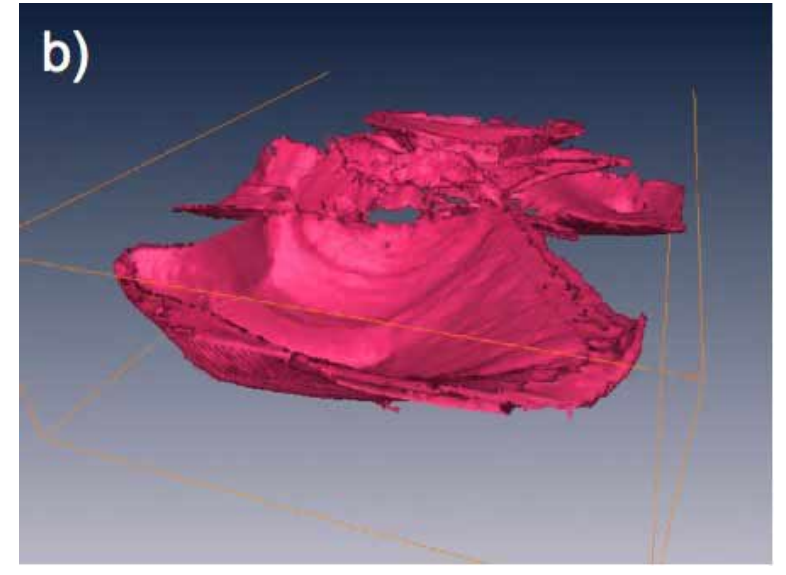

Figure 5. 3D representations of sub-surface cracking under a $350 \mathrm{mN}$ indent that underwent a giant pop-in. a) A portion of a lateral crack, superimposed on one of the cross-sections from the sequence. b) The full reconstructed crack system. c) Surface view of the indent.

\section{CONCLUSIONS}

A giant pop-in event was observed in the P-h curve of crystalline Ge under indentation. The pop-in is caused by material removal, which is in turn triggered by lateral crack-induced chipping on loading. Elbowing in the P-h curve occurs on unloading due to the opening of lateral cracks: the plates of material above the cracks act as cantilevers, giving a linear elastic response. Indents featuring a giant pop-in were observed by Raman and TEM to contain amorphous phases. The mechanism of amorphisation is yet to be determined. 


\section{REFERENCES}

1 J. E. Bradby, J. S. Williams, J. Wong-Leung, M. V. Swain, and P. Munroe, Appl. Phys. Lett. 80, 2651-2653 (2002).

2 S. Q. Xiao and P. Pirouz, Journal of Materials Research 7, 1406-1412 (1992).

3 A. Kailer, K. G. Nickel, and Y. G. Gogotsi, J. Raman. Spectr. 30, 939-946 (1999).

$4 \quad$ P. Lemaitre, Journal of Materials Science Letters 7, 895-896 (1988).

5 B. R. Lawn, B. J. Hockey, and S. M. Wiederhorn, Journal of Materials Science 15, 1207 1223 (1980).

6 J. E. Bradby, J. S. Williams, J. Wong-Leung, M. V. Swain, and P. Munroe, Appl. Phys. Lett. 77, 3749-3751 (2000).

7 D. R. Clarke, M. C. Kroll, P. D. Kirchner, R. F. Cook, and B. J. Hockey, Phys. Rev. Lett. 60, 2156-2159 (1988). 